\title{
Ultrafast force-clamp spectroscopy of single molecules reveals load dependence of myosin working stroke
}

\author{
Marco Capitanio ${ }^{1,2}$, Monica Canepari ${ }^{3}$, Manuela Maffei $^{3}$, Diego Beneventi ${ }^{1}$, Carina Monico ${ }^{1}$, Francesco Vanzi $^{1,4}$, \\ Roberto Bottinelli ${ }^{3,5} \&$ Francesco Saverio Pavone ${ }^{1,2,6,7}$
}

\begin{abstract}
We describe a dual-trap force-clamp configuration that applies constant loads between a binding protein and an intermittently interacting biological polymer. The method has a measurement delay of only $\sim 10 \mu \mathrm{s}$, allows detection of interactions as brief as $\sim 100 \mu$ s and probes sub-nanometer conformational changes with a time resolution of tens of microseconds. We tested our method on molecular motors and DNA-binding proteins. We could apply constant loads to a single motor domain of myosin before its working stroke was initiated $(0.2-1 \mathrm{~ms})$, thus directly measuring its load dependence. We found that, depending on the applied load, myosin weakly interacted (<1 ms) with actin without production of movement, fully developed its working stroke or prematurely detached ( $<5 \mathrm{~ms}$ ), thus reducing the working stroke size with load. Our technique extends single-molecule force-clamp spectroscopy and opens new avenues for investigating the effects of forces on biological processes.
\end{abstract}

Force has a fundamental role in a wide array of biological processes. For example, it modulates enzymatic activity, induces structural changes in proteins and nucleic acids, alters kinetics of molecular bonds ${ }^{1,2}$, regulates motion of molecular motors ${ }^{3,4}$, and has a role in mechanical transduction and sensory functions ${ }^{5}$. At the molecular level, these processes are ultimately related to the capacity of force to modulate lifetimes of molecular interactions and transition rates in biochemical reaction cycles that involve motion ${ }^{6}$.

Single-molecule force spectroscopy techniques such as atomic force microscopy, optical tweezers and magnetic tweezers have opened up the possibility of studying such fundamental processes at the molecular level ${ }^{7}$. Protocols for single-molecule force spectroscopy have been devised for the study of stable and longlived bonds between two molecules. Commonly, one of the two molecules (A) is bound to a glass slide, whereas the other (B) is bound to a force probe such as the probe tip in an atomic force microscope or a bead trapped in optical tweezers (Fig. 1a). A link between the two molecules is established by bringing them in close proximity, and the bond lifetime is measured as a function of the loading rate (dynamic force spectroscopy ${ }^{8,9}$ ) or under a constant force (force-clamp spectroscopy ${ }^{10-12}$ ). Such experimental configurations have been applied to the study of bond rupture forces in single ligand-receptor or antigen-antibody complexes ${ }^{13}$, processive molecular motors stepping along their track (myosin $\mathrm{V}$ on $\operatorname{actin}^{3}$, kinesin on microtubules ${ }^{14}$, and enzymes processing DNA and $\mathrm{RNA}^{15}$ ) as well as to the study of the folding of proteins or nucleic acids ${ }^{1}$. If one of the interacting molecules is an extended biological polymer, a double trap (or 'dumbbell') assay is usually used ${ }^{16}$ (Fig. 1b).

When a molecular bond is weak, however, the unbinding kinetics becomes rapid and application of such protocols during the short lifetime of the molecular complex becomes challenging. Such molecular interactions include receptors with lowbinding-affinity ligands, nonprocessive motors interacting with their tracks and nucleic acid-binding proteins interacting with nonspecific sequences during the target search. Different protein conformations usually have different binding strengths, some of them characterized by rapid unbinding kinetics. Molecular interactions on the millisecond time scale are very common, and single-molecule force spectroscopy of such short-lived molecular complexes requires sub-millisecond resolution and control of the applied load. However, detection of interactions and application of controlled forces on the sub-millisecond time scale face a fundamental challenge in single-molecule force spectroscopy.

Let us consider a typical single or double optical trap experiment (Fig. 1a,b). In both cases, the movement of the force probe (the trapped bead or dumbbell) is governed by thermal agitation, with position variance given by $\left\langle x^{2}\right\rangle=k_{\mathrm{B}} T / k$, where $k_{\mathrm{B}}$ is the Boltzmann constant, $T$ is the temperature and $k$ is the stiffness of the system. Binding and unbinding are usually detected from variations in $\left\langle x^{2}>\right.$ because $k$ is greatly increased upon binding (Fig. 1c). The capacity to detect binding events on a short time scale is thus governed by the frequency response of thermal noise, which limits the shortest detectable event to $\sim 5-10 \mathrm{~ms}$ in typical experimental configurations ${ }^{7,17}$ (Fig. 1d and Supplementary

\footnotetext{
${ }^{1}$ European Laboratory for Non-linear Spectroscopy, University of Florence, Sesto Fiorentino, Italy. ${ }^{2}$ Department of Physics and Astronomy, University of Florence, Sesto Fiorentino, Italy. ${ }^{3}$ Department of Molecular Medicine, University of Pavia, Pavia, Italy. ${ }^{4}$ Department of Evolutionary Biology, University of Florence, Florence, Italy. ${ }^{5}$ Fondazione Salvatore Maugeri, Scientific Institute of Pavia, Pavia, Italy. ${ }^{6}$ National Institute of Optics-National Research Council, Florence, Italy. ${ }^{7}$ International Center of Computational Neurophotonics, Sesto Fiorentino, Italy. Correspondence should be addressed to M. Capitanio (capitan@lens.unifi.it).

RECEIVED 1 DECEMBER 2011; ACCEPTED 6 AUGUST 2012; PUBLISHED ONLINE 2 SEPTEMBER 2012; D0I:10.1038/NMETH.2152
} 
Figure 1 | Present limits on time resolution owing to thermal noise. (a) Schematic of a single-trap configuration to study the interaction between two biological molecules (molecule A bound to the cover slip; molecule B bound to a bead trapped in optical tweezers). (b) Schematic of a dual-trap or dumbbell configuration. $B$ can be actin, a microtubule, DNA, RNA or any polymer and A, any protein interacting with B (myosin, kinesin, dynein, DNA or RNA-binding proteins). (c) Drawing of the position signal of a trapped bead while molecules $A$ and $B$ are interacting. As the stiffness of the molecular complex A-B $\left(k_{b}\right)$ is usually much higher than the stiffness of the optical tweezers $\left(k_{u}\right)$, a sharp reduction in position noise is observed upon bond formation. (d) For a bead trapped in optical tweezers, power spectra of position noise in unbound and bound states decrease for frequencies above their respective cut-off frequencies $f_{\mathrm{C}}{ }^{\mathrm{u}}$ and $f_{\mathrm{C}}{ }_{\mathrm{C}}^{\mathrm{b}}$. As the cut-off frequency is proportional to the system stiffness and $k_{\mathrm{b}}>k_{\mathrm{u}} \rightarrow f_{\mathrm{C}}^{\mathrm{b}}>f_{\mathrm{C}}^{\mathrm{u}}$. The area under the power spectrum for frequencies greater than $f$ (shaded areas) is the position variance $<x^{2}>$ measured on finite time interval $\Delta t=1 / f$. Reduction of $<x^{2}>$ upon bond formation is detectable only for frequencies $f<<f_{C}$, limiting the shortest detectable event to $\sim 5 \mathrm{~ms}$ under optimal experimental conditions of current systems based on thermal noise reduction.
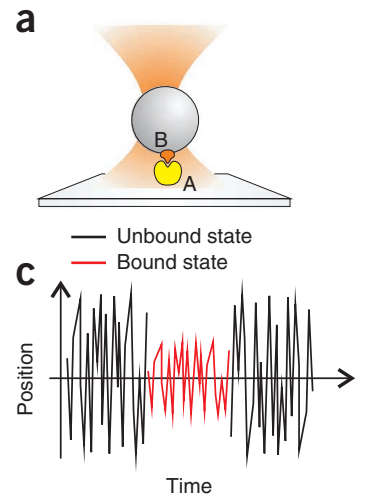

b

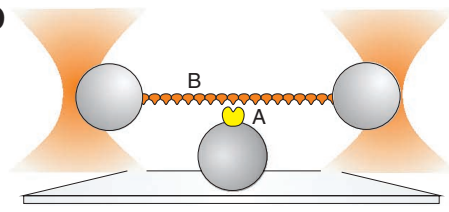

d

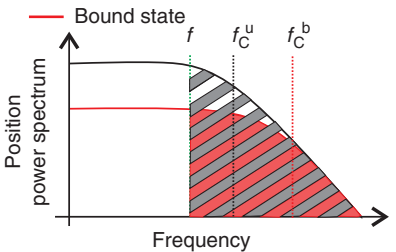

- Unbound state
Fig. 1). Moreover, as load is usually applied after detection of the molecular-bond formation, load cannot be applied until several milliseconds after binding ${ }^{18}$.

We developed an ultrafast force-clamp spectroscopy technique that uses a dual trap configuration to apply constant loads between a single intermittently interacting biological polymer and a binding protein. Our system has a delay of only $~ 10 \mu$ s between formation of the molecular bond and application of the force, and can detect interactions as short as $100 \mu \mathrm{s}$. The force-clamp configuration in which our assay operates allows direct measurements of the load dependence of the lifetimes of single molecular bonds. Moreover, conformational changes of single proteins and molecular motors can be recorded with sub-nanometer accuracy and a temporal resolution in the tens of microseconds. We tested our technique on molecular motors, using myosin II from fast skeletal muscle, and on a protein-DNA interaction, for the lactose repressor (LacI) interaction with DNA.

Skeletal muscle myosin is a nonprocessive molecular motor characterized by a very rapid enzymatic activity. Muscle performance at the molecular level is determined by the elementary displacement (working stroke) produced by the motor protein myosin II and its dependence on load, but the molecular mechanism underlying load dependence of the myosin working stroke remains unclear. In fact, the very fast development of the working stroke by skeletal muscle myosin, which is concluded about one millisecond after actin-myosin attachment ${ }^{19}$, has not allowed such measurement up to now ${ }^{16,20}$. Using our method, we applied controlled loads to a single myosin head before the working stroke initiated, thus directly measuring its load dependence. This revealed previously hidden sub-millisecond interaction pathways and disclosed the molecular mechanism of load dependence of the myosin working stroke, reconciling the apparently contradictory results found in the literature ${ }^{4,21}$.

For the interaction of LacI with DNA, we dissected the DNA sequence- and force-dependent kinetics of LacI binding to DNA at high temporal resolution and detected differences between specific (operator) and nonspecific sequences. These results suggest the method will be useful for investigating the fast target-search mechanisms of a variety of DNA-binding and DNA-processing proteins and the possible conformational changes that occur upon recognition of the target.

\section{RESULTS}

\section{Ultrafast force-clamp spectroscopy}

A sketch of the operational principle of our method is shown in Figure 2a. The polymer B (here actin or DNA) is first stretched between two optically trapped beads and brought in close proximity of a third bead, where the molecule A (here myosin or LacI) is present. In this configuration, equal and opposite forces are applied to left and right beads $\left(F_{\mathrm{L}}=-F\right.$ and $\left.F_{\mathrm{R}}=+F\right)$ and the net force $\left(F_{\text {tot }}\right)$ applied to the polymer is zero ${ }^{16}$ (Supplementary Fig. 2). Second, the force applied by each trap is clamped to two different values, $F_{\mathrm{L}}=-F$ and $F_{\mathrm{R}}=+F+\Delta F$, so that the net force applied to the dumbbell becomes $F_{\text {tot }}=+\Delta F$. The forces acting on the two beads are clamped independently by a doublefeedback system that moves the traps using acousto-optic deflectors (AODs) to keep the forces constant. Because a force, $F_{\text {tot }}$, is applied to the dumbbell, when molecules A and B are not bound, the dumbbell moves against viscous drag at constant velocity, $v=$ $F_{\text {tot }} / \gamma$ ( $\gamma$ is the viscous drag coefficient of the dumbbell). When the displacement of the dumbbell exceeds a preset value (typically 100-200 $\mathrm{nm}$ in our experiments), the force applied by the traps is reversed $\left(F_{\text {tot }}=-\Delta F\right)$, to maintain the two molecules in proximity of each other. The dumbbell, thus, moves in the opposite direction until it reaches the initial position, and the force is switched again to $F_{\text {tot }}=+\Delta F$. Therefore, when the two molecules are not bound, position of the traps over time is a triangular wave (Fig. 2b). When the molecular bond is formed, $F_{\text {tot }}$ is transferred to molecule $\mathrm{A}$, which is bound to the cover slip; as the system was designed to maintain a constant force, the dumbbell suddenly stops. Therefore, the position signal of the traps becomes a flat line (except for possible conformational changes of the molecules after binding, such as a $\sim 5 \mathrm{~nm}$ movement owing to the myosin working stroke, as shown below).

Our experimental scheme has two major advantages compared to previous ones. First, $F_{\text {tot }}$ is always applied to molecule B, so that molecule A very rapidly experiences such force upon binding. The time $(\tau)$ taken to transfer $F_{\text {tot }}$ from the viscous solution to molecule A and stop the dumbbell depends predominantly on the stiffness of the molecular complex and on the viscous drag coefficient of the dumbbell. This time is very short compared to the duration of usual protein interactions ( $\tau$ of $\sim 10 \mu$ s for the actinmyosin complex; Supplementary Note 1). Therefore, molecular 
a
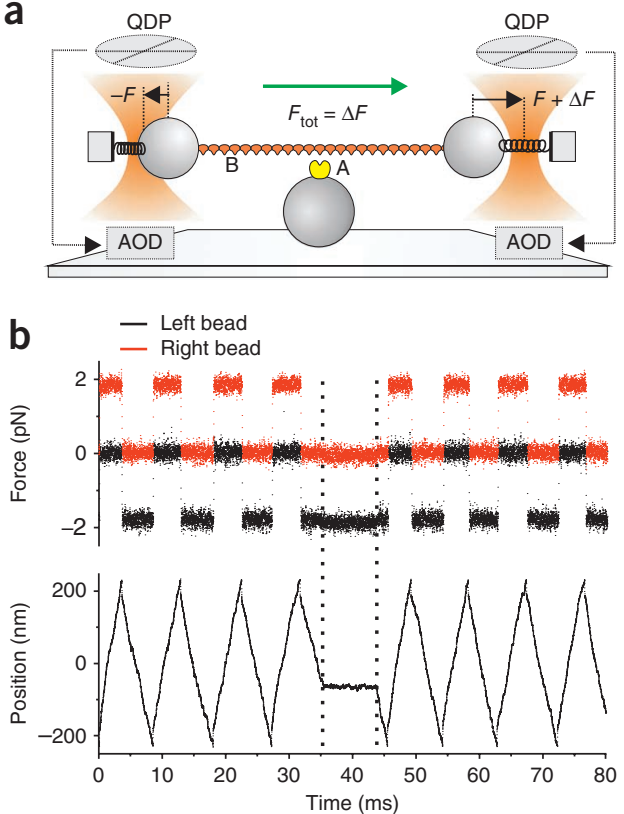

C

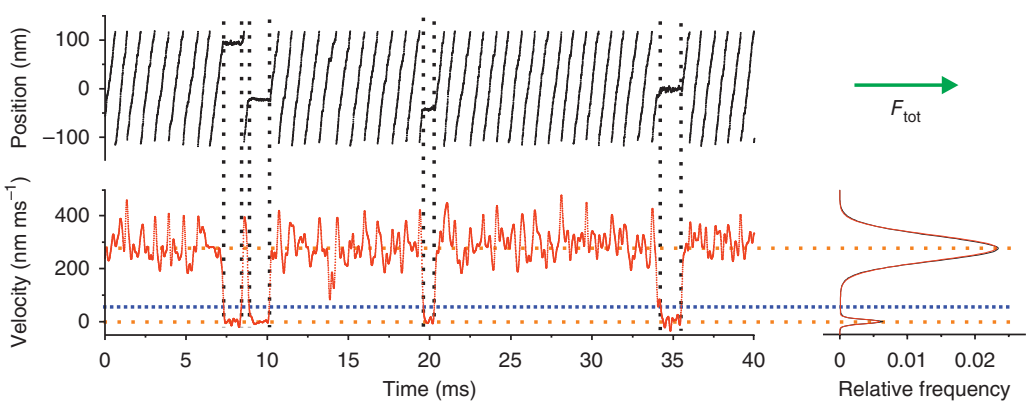

d

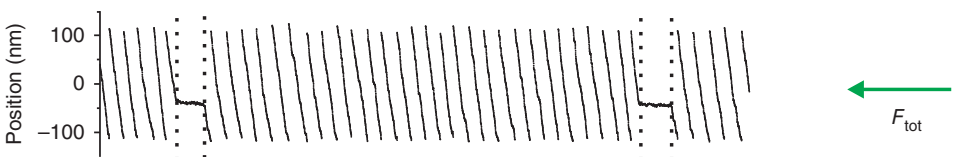

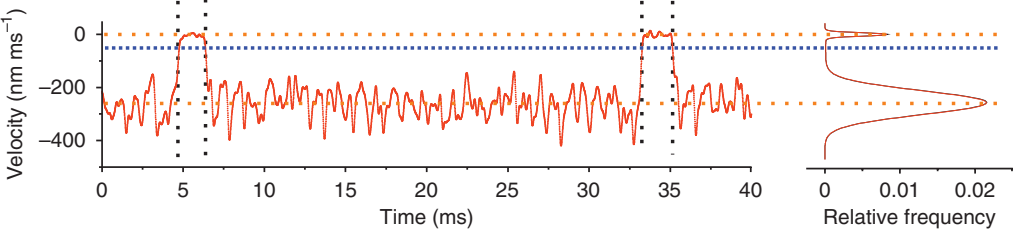

Figure 2 | Ultrafast force-clamp spectroscopy. (a) Schematic of operational principle of our method illustrating constant $F_{\text {tot }}=\Delta F$ applied to molecule $\mathrm{B}$ through two feedback systems clamping the force on the left and right bead to $-F$ and $F+\Delta F$, respectively. The force is measured using quadrant detector photodiodes (QDPs) and kept constant by moving the traps through AODs. (b) $\Delta F$ on left and right beads (top). Position of one of the two traps (bottom). The force is switched between $+\Delta F$ and $-\Delta F$ to keep the dumbbell within a confined spatial interval $( \pm 200 \mathrm{~nm})$. The dumbbell stops when $\mathrm{A}$ binds to $\mathrm{B}$ (dotted lines). (c, d) Position signals under constant positive (c) and negative (d) force (top) and dumbbell velocity (bottom). Velocity distribution (right) perfectly fits the sum of two Gaussian functions, one centered on zero (bound state), the other one centered on $v=F_{\text {tot }} / \gamma$ (unbound state). Binding and unbinding events (black dotted lines) were detected with a threshold (blue dotted line) chosen to assure $<1 \%$ false events (Online Methods). Data in $\mathbf{b}-\mathbf{d}$ are from actin-myosin experiments.

interactions occur under a real force-clamp configuration, allowing, for example, a direct measurement of molecular complex lifetimes as a function of force. In the case of actin and myosin, the working stroke is performed under a constant load, as demonstrated below.

The second advantage of our system is that bound and unbound states can be detected through the derivative of the position signal (the dumbbell velocity), as the dumbbell velocity drops to zero upon binding (Fig. 2c,d, where we separated signals under positive and negative forces for independent analysis). Velocity changes occur with the same very short time constant, $\tau$; but to increase the signal-to-noise ratio of the dumbbell velocity and prevent detection of false events we time-averaged velocity data, thus reducing the capability to detect very short interactions. We detected bound and unbound events as short as $100 \mu \mathrm{s}$ and $20 \mu$ s, respectively (Supplementary Notes $\mathbf{2}-\mathbf{5}$ ). These values are still about two orders of magnitude smaller than the typical limit of $\sim 5-10$ ms of previous techniques, in which detection of the interaction is based on a reduction in Brownian noise ${ }^{17}$.

In typical traces obtained applying our method to actin-myosin and DNA-LacI interaction (Fig. 3), thanks to the stabilization of our apparatus to less than $1 \mathrm{~nm}^{22,23}$, we discerned specific binding regions along the actin filament and DNA molecule. Owing to the helical pitch of the actin filament, only a few actin monomers a
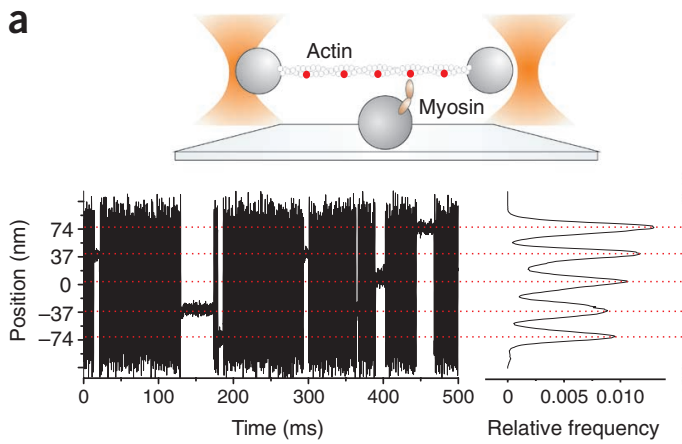

b

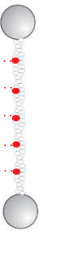

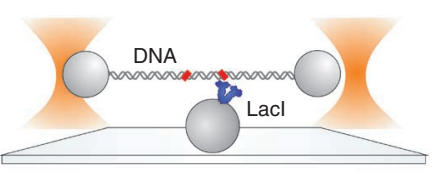

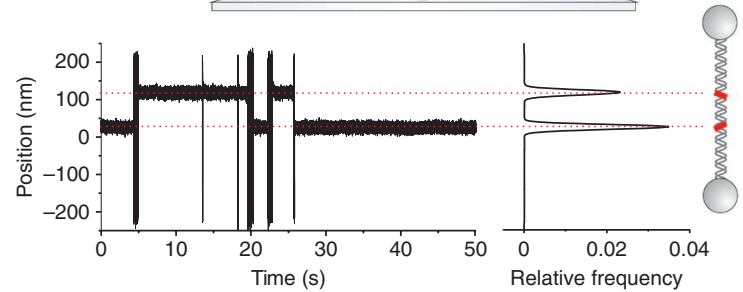

Figure 3 | Implementation of ultrafast force-clamp spectroscopy on different biological systems. (a) Typical record of a single myosin molecule interacting with an actin filament ([ATP] $=50 \mu \mathrm{M}, F_{\text {tot }}=7 \mathrm{pN}, \pm 100 \mu \mathrm{m}$ confined dumbbell oscillation; left). Distribution of bound positions (right) shows peaks corresponding to the helix periodicity of the actin filament $(\sim 37 \mathrm{~nm})$. (b) Typical record of a LacI molecule interacting with a DNA molecule $\left(F_{\text {tot }}=5 \mathrm{pN}, \pm 200 \mu \mathrm{m}\right.$ confined dumbbell oscillation; left). Distribution of bound positions (right) shows peaks separated by the distance ( 96 nm) between the two LacI operators (red) in the DNA molecule. The triangular wave (visible in Fig. $\mathbf{2} \mathbf{b}$ ), is too fast to be visible on the time scale displayed here. Drawings are not to scale. 

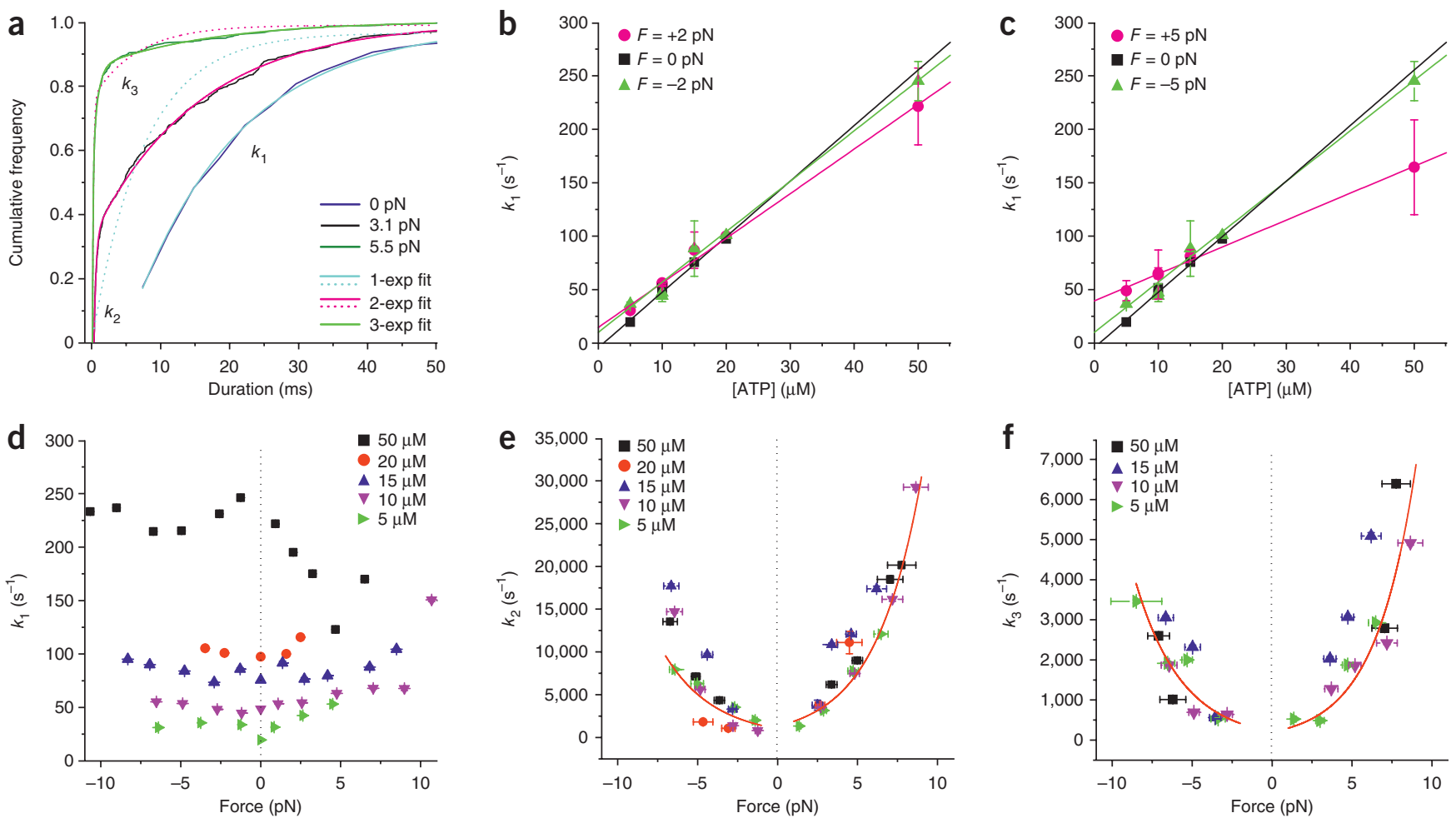

Figure 4 | Load-dependent kinetics of the actin-myosin interaction dissected with microsecond time resolution. (a) Typical cumulative frequency distributions of the duration of $n$ bound-state events with $10 \mu \mathrm{M}$ ATP for forces of $0 \mathrm{pN}(n=37),+3.1 \mathrm{pN}(n=215)$ and $+5.5 \mathrm{pN}(n=329)$. The distributions show a single-exponential fit (1-exp fit) under zero force, two-exponential fit (2-exp fit) under low force and three-exponential fit (3-exp fit) under high force. $(\mathbf{b}, \mathbf{c}) k_{1}$ versus [ATP] at inidicated forces. (d) $k_{1}$ versus force for indicated [ATP]. (e,f) $k_{2}$ and $k_{3}$ versus force for indicated [ATP]. Red curve is the Bell-bond weighted fitting curve through all data $k_{2,3}(F)=k_{2,3}(0) \times \exp \left(\alpha_{2,3} \times F / k_{\mathrm{B}} T\right)\left(F>0: k_{2}(0)=1,320 \pm 140 \mathrm{~s}^{-1}, \alpha_{2}=1.42 \pm\right.$ $\left.0.07 \mathrm{~nm} ; k_{3}(0)=200 \pm 25 \mathrm{~s}^{-1}, \alpha_{3}=1.60 \pm 0.09 \mathrm{~nm} ; F<0: k_{2}(0)=1,020 \pm 170 \mathrm{~s}^{-1}, \alpha_{2}=-1.3 \pm 0.1 \mathrm{~nm} ; k_{3}(0)=210 \pm 60 \mathrm{~s}^{-1}, \alpha_{3}=-1.4 \pm 0.2 \mathrm{~nm}\right)$. Data are for $n=36$ (b) and $n=39$ (c) measurements on 6 molecules, each comprising several hundreds of interactions, and for $n=173$ (d-f) measurements on 7 molecules. Error bars, s.e.m. Error bars in $\mathbf{d}$ are smaller than the symbols.

every $\sim 37 \mathrm{~nm}$ were properly oriented for optimal interaction with myosin, thus modulating the binding of myosin along actin (Fig. 3a). In contrast, Lac repressor acts by binding to its specific target (the operator sequence) in the lac operon to prevent transcription of the downstream genes coding for the proteins involved in lactose uptake and metabolism. Our DNA construct comprised two O1 operators spaced 305 base pairs apart ( $96 \mathrm{~nm}$ under our experimental conditions). LacI strongly bound to these regions (Fig. 3b). The method enabled measurements of the dependence of bound-state durations for LacI interacting with different sequences and subject to various forces (Supplementary Results). This type of measurement can provide new information, for example, on the mechanisms of target search on DNA, on the force-dependence of protein-DNA interactions and gene regulation, and on the fast kinetics possibly involved in genetic noise and stochastic transcription events. We discuss some of these aspects specifically related to LacI in Supplementary Results.

\section{Sub-millisecond kinetics of myosin under load}

Using our method, we analyzed the actin-myosin interaction in detail. We first collected data on each myosin molecule under zero force; in this case, detection of interactions was based on Brownian noise and was thus affected by a limited time resolution $(\sim 5-10 \mathrm{~ms})^{16,19}$. Using the same molecule we then applied our ultrafast force-clamp assay and acquired data under forces ranging from $\pm 1 \mathrm{pN}$ to $\pm 10 \mathrm{pN}$. We first analyzed the duration distributions of bound-state events under different forces and ATP concentrations (Fig. 4). We defined positive and negative forces as forces respectively opposing or assisting the myosin working stroke, which we determined under zero force ${ }^{19,24}$.

Measurements performed at zero force always showed a single exponential distribution (Fig. 4a). The detachment rate $\left(k_{1}\right)$ was linearly dependent on ATP concentration in the 5-20 $\mu \mathrm{M}$ range, with a second-order rate constant of $(5.2 \pm 0.3) \times 10^{6} \mathrm{M}^{-1} \mathrm{~s}^{-1}$, consistent with previous results ${ }^{19}$ (Fig. $4 \mathrm{~b}$ ). When we switched to our force-clamp assay with increased time resolution, we uncovered a second population of much shorter events, clearly highlighted by a bi-exponential distribution comprising a second and much faster rate, $k_{2}$ (Fig. 4a). $k_{2}$ was highly variable with load, the dependence of $k_{2}$ on force being well fitted by an exponential Bell-bond curve, with slightly higher rates for positive forces (Fig. 4e). Experiments performed with 5-50 $\mu \mathrm{M}$ ATP did not show a dependence of $k_{2}$ on [ATP]. In contrast, the behavior of the slower $k_{1}$ at low forces $(F= \pm 2 \mathrm{pN})$ was superimposable with that obtained at zero force, linearly dependent on [ATP] in the 5-50 $\mu \mathrm{M}$ range, with a second-order rate constant of $(4.2 \pm 0.2) \times 10^{6} \mathrm{M}^{-1} \mathrm{~s}^{-1}$ and $(4.7 \pm 0.3) \times 10^{6} \mathrm{M}^{-1} \mathrm{~s}^{-1}$ for positive and negative forces, respectively (Fig. $\mathbf{4 b}, \mathbf{d}$ ). As we increased the force, $k_{1}$ was only minimally affected by negative loads (Fig. $4 c ; F=-5 \mathrm{pN}$ ). In contrast, $k_{1}$ showed a slight increase for positive forces at low [ATP] 
Figure 5 | Measuring conformational changes with sub-nanometer spatial and microsecond temporal resolution: working stroke of skeletal muscle myosin under force. (a) Long (>1 ms) single actin-myosin interactions under resistive load. Magenta arrowheads indicate actin-myosin binding, green arrowheads myosin working stroke and cyan arrowheads actin-myosin detachment. (b) Short events (<1 ms) under resistive force. (c) Ensemble average of 191 events with durations $>1.5 \mathrm{~ms}$ under $+3.2 \mathrm{pN}$ force. $t=0$ is time of binding. Before binding, the dumbbell moved at constant velocity against viscous drag $\left(v=-178 \mathrm{~nm} \mathrm{~ms}^{-1}\right)$. After binding, myosin produced a $\sim 4 \mathrm{~nm}$ working stroke in the opposite direction in few milliseconds. Development of the working stroke was fitted by a bi-exponential function (magenta curve) (Supplementary Note 7). (d) Analysis of short events $(<1.5 \mathrm{~ms}, n=131)$ and long events $(>1.5 \mathrm{~ms}, n=191)$.

(where myosin is bound to actin prevalently in the rigor state, that is, with no nucleotide bound) and a decrease at high [ATP] (where the myosin-ADP state is more populated; Fig. 4c; $F=+5$ $\mathrm{pN}$ ). Our data suggest that detachment of myosin in the rigor state is accelerated by a resistive force, as observed in ref. 25 , whereas ADP release is slowed down, as it is usually assumed in models of muscle contraction ${ }^{26,27}$. Experiments at very low and saturating ATP concentrations would be required to assess this thesis.

As we increased the force, fitting of the event duration distribution with a double exponential became less accurate, necessitating a third exponential in the fitting function (Fig. 4a). This suggests that, as the force is increased, a third population of unbinding events becomes more probable. The detachment rate of this third population of events $\left(k_{3}\right)$ had characteristics similar to those of $k_{2}$, being ATP-independent and following a Bell-bond curve against force, with slightly higher rates for positive forces but $k_{3}$ was $\sim 5$ times slower than $k_{2}$ and faster than $k_{1}$ (Fig. 4f). These three load-dependent detachment pathways correspond to different conformations of the actin-myosin complex, as disclosed by the analysis of the myosin mechanics reported below.

\section{Development of the myosin working stroke under load}

Close inspection of single actin-myosin interactions showed that, under resistive loads, a 5-10 $\mathrm{nm}$ stroke usually followed binding after a short dwell time $(0.2-1 \mathrm{~ms}$; Fig. 5a). However, some events, especially short events $(<1 \mathrm{~ms})$, did not exhibit any stroke (Fig. 5b). To understand how the myosin working stroke is developed along the three observed detachment pathways, we separated the events belonging to the three populations based on their duration (Online Methods). To reduce position noise, we separately evaluated the myosin working stroke for each population of events in a record by ensemble-averaging the interactions. This procedure allowed us to attain sub-nanometer spatial resolution with a temporal resolution of tens of microseconds (Supplementary Notes 6,7). In Figure 5c we show an ensemble average under resisting force $(F=+3.2 \mathrm{pN})$, from which we excluded the very short events $\left(k_{2}\right)$ : before binding (time $\left.(t)<0\right)$, the actin filament is moving at constant velocity; after binding, the filament stops and then starts moving in the opposite direction, driven by the myosin working stroke. Under assisting force, the working stroke developed very rapidly after binding and in the same direction of the dumbbell movement before binding (Supplementary Fig. 3); for these reasons, the separation of the two movements and the quantification of the working stroke amplitude were not always possible. Thus, here we limited our analysis to resistive forces, which we compared with previous literature ${ }^{4,21}$.
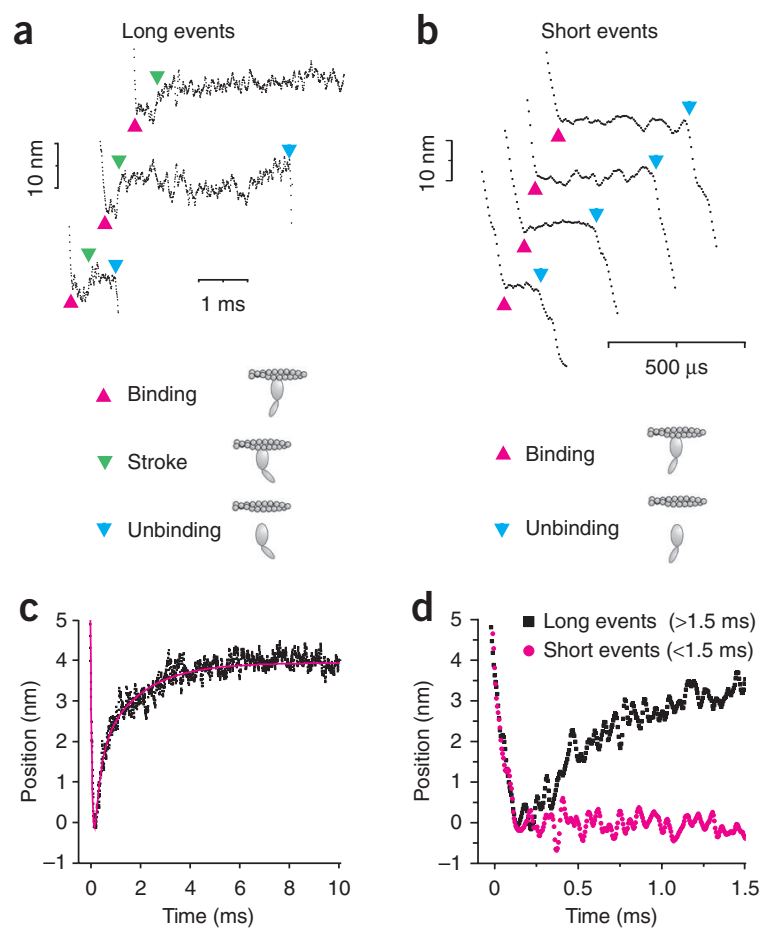

The movement produced by myosin turned out to be very different for the three classes of events. Very short events $\left(k_{2}\right)$ did not produce any working stroke, independently of the force applied (Fig. 5d). In contrast, when we excluded the very short events from the ensemble average, the working stroke $(d)$ was different from zero and decreased with force from $d_{0}$ (unloaded working stroke) to 0 , when a critical force $F_{0}$ was reached (the isometric force) (Fig. 6a). The average value of the isometric force was $5.7 \pm$ $0.6 \mathrm{pN}$ (mean \pm s.e.m. of 6 molecules). The average value of the unloaded working stroke was $5.0 \pm 0.6 \mathrm{~nm}$ (mean \pm s.e.m. of 5 molecules), consistent with previous single-molecule experiments in which myosin was randomly attached to the platform bead ${ }^{19}$ and on the lower bound of what has been observed in other systems ${ }^{6}$. Finally, when we averaged only the long events $\left(k_{1}\right)$, excluding both the very short $\left(k_{2}\right)$ and intermediate events $\left(k_{3}\right)$, the amplitude of the working stroke was constant, independent of the force applied. When the isometric force was reached, the working stroke suddenly dropped to zero (Fig. 6a).

Collectively, kinetics and mechanical data provided insight into the regulation of the actinomyosin cycle and the working stroke by force. As $k_{1}$ is the only ATP-dependent rate, $k_{1}$ must be the rate of detachment of myosin from actin after ATP binding, that is, at the end of the cycle and after the working stroke has been completed (Fig. 6b). Indeed, ensemble averages of long events showed that the working stroke was always fully developed under forces below the isometric force. In contrast, $k_{2}$ must be the rate of detachment of myosin from actin at the very beginning of the cycle. In fact, $k_{2}$ was very fast and ATP-independent. Moreover, analysis of the mechanics demonstrated that such short events do not produce any working stroke. We hypothesize that $k_{2}$ is the detachment rate of myosin in the weak binding state 28,29 , that is, with ADP and inorganic phosphate still bound (before producing any working stroke; Fig. 6b). Recently, such a state has been reported in studies performed on single myosin VI molecules, observing similar detachment rates ${ }^{30}$. Also $k_{3}$ was ATP-independent; 
Figure 6 | Load dependence of myosin working stroke. (a) Working stroke $d$, normalized to the unloaded working stroke $d_{0}$, versus force, normalized to the isometric force $F_{0}$. Working stroke size was obtained from ensemble averages excluding very short events $\left(k_{2}\right)$ (black), and excluding both short $\left(k_{2}\right)$ and intermediate events $\left(k_{3}\right)$ (including long events, $k_{1}$, only; red). Data are from 43 measurements, each comprising several hundreds of interactions, grouped by similar force and averaged. Error bars, s.e.m.

(b) Model of actin-myosin interaction.

$k_{1}$ is the detachment rate at the end of the cycle, induced by ATP binding, after completion of a $\sim 5-n m$ working stroke; $k_{2}$ is the detachment rate of the weak binding state (myosin and ADP-inorganic phosphate $\left(P_{i}\right)$ ), at the beginning of the cycle, without production of any stroke; $k_{3}$ is a premature detachment in a ADP or ADP- $P_{i}$ strong binding state, induced by force before the completion of the working stroke. (c) Ratio between the number of premature unbinding events $\left(A_{3}\right)$ and the number of long events completing the working stroke $\left(A_{1}\right)$ versus force. Data are from 17 measurements, each comprising several hundreds of interactions, grouped by similar force and averaged. Error bars, s.e.m.

therefore, detachment must happen before ATP binding, and inclusion of such events in the ensemble averages progressively reduced the working stroke amplitude with force. Such events should, thus, represent a premature unbinding of myosin from actin (in the ADP or in the ADP and inorganic phosphate strongbinding state) before the completion of the working stroke. In fact, as we increased the force, the rate of premature detachment $k_{3}$ increased, thus decreasing the population of $k_{1}$ events (Fig. 6c). As only the latter events are associated with the production of a full working stroke, the average amplitude of the working stroke decreased with force.

\section{DISCUSSION}

Several experimental configurations have been developed in the past to investigate load dependence of short-lived molecular complexes ${ }^{18,30}$, but each has limitations. The technique developed in ref. 18 is limited by a delay of several milliseconds between binding and application of the load, can only be used to detect interactions longer than one millisecond and cannot be used to dynamically follow force variations. In ref. 30 , a single optical trap rapidly moving in alternating directions had been used to detect interactions based on the trap position rather than from Brownian noise, thus detecting sub-millisecond events, but it was limited to a constant velocity rather than a constant force configuration, and measurement of conformational changes of the interacting molecules was not possible. To our knowledge, our methodology is unique in combining 'instantaneous' application and clamping of the force, detection of molecular interactions as short as $\sim 100 \mu$ s and the capability to probe sub-nanometer conformational changes occurring during the interaction on a time scale of a few tens of microseconds.

Our method revealed a complex mechanism of regulation of the myosin working stroke by force and previously undetectable fast detachment pathways. In particular, a premature $(<5 \mathrm{~ms})$ dissociation pathway becomes more populated as the force is increased, resulting in a working stroke that decreased with load. Our data suggest that the decrease in the amplitude of the working stroke observed in muscle fibers ${ }^{4,31}$ is due to premature unbinding of the molecule from actin, thus providing a molecular explanation for the mechanism of regulation of the myosin working stroke in the intact muscle. Experiments conducted in ref. 21 on small arrays of myosin molecules reported a load-independent size of the working stroke that seemed to contradict the prior results obtained on muscle fibers. That result is in agreement with the constant working stroke we found when averaging only the long events and is probably a consequence of the inability to detect premature detachment events in those experiments ${ }^{21}$ owing to limited time resolution and a different experimental configuration. Our data therefore reconcile the contradictory results found in the literature.

Our preliminary results on the Lac repressor-DNA interaction showed two different kinds of interactions: long-lived interactions targeting the operator sequence of the lac operon and short-lived interactions occurring everywhere along the DNA molecule. Such fast interactions are probably involved in the facilitated diffusion mechanism that is expected to increase the binding rate of LacI to the operator sequences ${ }^{32}$.

In its present implementation (dumbbell assay), our methodology could be applied in a straightforward fashion to analyze various nonprocessive motors, single domains of processive motors, and protein-DNA and protein-RNA interactions. By exchanging the actin filament with a microtubule, as in ref. 33, our technique could be easily applied to nonprocessive kinesins (NCD, KIF1D and others) or single motor domains of processive motors belonging to the kinesin and dynein family. The potential applications of this method to DNA-protein interactions range from motors that process along a nucleic acid (for example, DNA and RNA polymerases ${ }^{15}$, helicases and translocases $^{34,35}$ ) to transcription factors and a wide variety of DNAbinding proteins. The advantages of our method can be extended to these proteins, providing improved temporal resolution for the study of the mechanism of target search ${ }^{32}$, the possible conformational changes occurring upon target recognition ${ }^{36}$, assembly of other subunits occurring at the target ${ }^{37}$, transitions 
occurring in the initiation phase of processive activity ${ }^{38,39}$ or in response to specific regulatory sequences ${ }^{34,35}$.

Finally, the same concept could be readily implemented in the simpler single-bead configuration (Fig. 1a), conceivably extending our technique to any short-lived protein-protein interaction.

\section{METHODS}

Methods and any associated references are available in the online version of the paper.

Note: Supplementary information is available in the online version of the paper.

\section{ACKNOWLEDGMENTS}

We thank G. Belcastro for his help with Lac repressor experiments, M. Giuntini for quadrant detector photodiode electronics, and V. Lombardi and L. Gardini for discussion. This research was funded by the EU Seventh Framework Programme (FP7/2007-2013; grant agreements B0 211383, B0 228334 and B0 241526), by the Italian Ministry of University and Research (PRIN 2006 2006051323_003, FIRB 2011 RBAP11X42L006 and Flagship Project NANOMAX) and by Ente Cassa di Risparmio di Firenze to F.S.P. and by the EU Seventh Framework Programme (FP7/2007-2013; grant agreement 223576, Combating age-related muscle weakness (MYOAGE)) to R.B.

\section{AUTHOR CONTRIBUTIONS}

M. Capitanio conceived and designed the ultrafast force clamp, performed experiments, analyzed data and wrote the paper. M.M. prepared samples and contributed to myosin experiments. D.B. contributed to setting up the ultrafast force clamp and performing myosin experiments. C.M. prepared samples and performed LacI experiments. F.V. supervised LacI experiments and wrote the paper. R.B. and M. Canepari supervised myosin experiments. R.B. contributed to writing the paper. F.S.P. supervised the design of the ultrafast force-clamp experiments and the whole project.

\section{COMPETING FINANCIAL INTERESTS}

The authors declare no competing financial interests.

Published online at http://www.nature.com/doifinder/10.1038/nmeth.2152 Reprints and permissions information is available online at http://www.nature. com/reprints/index.html.

1. Cecconi, C., Shank, E.A., Bustamante, C. \& Marqusee, S. Direct observation of the three-state folding of a single protein molecule. Science 309, 2057-2060 (2005).

2. Marshall, B.T. et al. Direct observation of catch bonds involving celladhesion molecules. Nature 423, 190-193 (2003).

3. Rief, M. et al. Myosin-V stepping kinetics: a molecular model for processivity. Proc. Natl. Acad. Sci. USA 97, 9482-9486 (2000).

4. Reconditi, M. et al. The myosin motor in muscle generates a smaller and slower working stroke at higher load. Nature 428, 578-581 (2004).

5. Laakso, J.M., Lewis, J.H., Shuman, H. \& Ostap, E.M. Myosin I can act as a molecular force sensor. Science 321, 133-136 (2008).

6. Howard, J. Mechanics of Motor Proteins and the Cytoskeleton. (Sinauer Associates, Inc., 2001)

7. Neuman, K.C. \& Nagy, A. Single-molecule force spectroscopy: optical tweezers, magnetic tweezers and atomic force microscopy. Nat. Methods 5 , 491-505 (2008).

8. Hinterdorfer, P. \& Dufrene, Y.F. Detection and localization of single molecular recognition events using atomic force microscopy. Nat. Methods 3, 347-355 (2006)

9. Guo, B. \& Guilford, W.H. Mechanics of actomyosin bonds in different nucleotide states are tuned to muscle contraction. Proc. Natl. Acad. Sci. USA 103, 9844-9849 (2006).

10. Wiita, A.P. et al. Probing the chemistry of thioredoxin catalysis with force. Nature 450, 124-127 (2007).

11. Lang, M.J., Asbury, C.L., Shaevitz, J.W. \& Block, S.M. An automated twodimensional optical force clamp for single molecule studies. Biophys. J. 83, 491-501 (2002).

12. Greenleaf, W.J., Woodside, M.T., Abbondanzieri, E.A. \& Block, S.M. Passive all-optical force clamp for high-resolution laser trapping. Phys. Rev. Lett. 95, 208102 (2005).
13. Litvinov, R.I., Shuman, H., Bennett, J.S. \& Weisel, J.W. Binding strength and activation state of single fibrinogen-integrin pairs on living cells. Proc. Natl. Acad. Sci. USA 99, 7426-7431 (2002).

14. Visscher, K., Schnitzer, M.J. \& Block, S.M. Single kinesin molecules studied with a molecular force clamp. Nature 400, 184-189 (1999).

15. Abbondanzieri, E.A., Greenleaf, W.J., Shaevitz, J.W., Landick, R. \& Block, S.M. Direct observation of base-pair stepping by RNA polymerase. Nature 438 460-465 (2005).

16. Finer, J.T., Simmons, R.M. \& Spudich, J.A. Single myosin molecule mechanics: piconewton forces and nanometer steps. Nature 368, 113-119 (1994).

17. Smith, D.A., Steffen, W., Simmons, R.M. \& Sleep, J. Hidden-Markov methods for the analysis of single-molecule actomyosin displacement data: the variance-Hidden-Markov method. Biophys. J. 81, 2795-2816 (2001).

18. Veigel, C., Molloy, J.E., Schmitz, S. \& Kendrick-Jones, J. Load-dependent kinetics of force production by smooth muscle myosin measured with optical tweezers. Nat. Cell Biol. 5, 980-986 (2003).

19. Capitanio, M. et al. Two independent mechanical events in the interaction cycle of skeletal muscle myosin with actin. Proc. Natl. Acad. Sci. USA 103, 87-92 (2006).

20. Takagi, Y., Homsher, E.E., Goldman, Y.E. \& Shuman, H. Force generation in single conventional actomyosin complexes under high dynamic load. Biophys. J. 90, 1295-1307 (2006).

21. Kaya, M. \& Higuchi, H. Nonlinear elasticity and an 8-nm working stroke of single myosin molecules in myofilaments. Science 329, 686-689 (2010).

22. Capitanio, M., Cicchi, R. \& Pavone, F.S. Position control and optical manipulation for nanotechnology applications. Eur. Phys. J. B 46, 1-8 (2005).

23. Capitanio, M., Maggi, D., Vanzi, F. \& Pavone, F. Fiona in the trap: the advantages of combining optical tweezers and fluorescence. J. Opt. A 9, S157 (2007).

24. Molloy, J.E., Burns, J.E., Kendrick-Jones, J., Tregear, R.T. \& White, D.C.S. Movement and force produced by a single myosin head. Nature 378, 209-212 (1995).

25. Lewalle, A., Steffen, W., Stevenson, 0., Ouyang, Z. \& Sleep, J. Singlemolecule measurement of the stiffness of the rigor myosin head. Biophys. J. 94, 2160-2169 (2008).

26. Nyitrai, M. \& Geeves, M.A. Adenosine diphosphate and strain sensitivity in myosin motors. Phil. Trans. R. Soc. Lond. B 359, 1867-1877 (2004).

27. Dantzig, J.A., Hibberd, M.G., Trentham, D.R. \& Goldman, Y.E. Cross-bridge kinetics in the presence of MgADP investigated by photolysis of caged ATP in rabbit psoas muscle fibres. J. Physiol. (Lond.) 432, 639-680 (1991).

28. Brenner, B. Rapid dissociation and reassociation of actomyosin crossbridges during force generation: a newly observed facet of cross-bridge action in muscle. Proc. Natl. Acad. Sci. USA 88, 10490-10494 (1991).

29. Geeves, M.A. The dynamics of actin and myosin association and the crossbridge model of muscle contraction. Biochem. J. 274, 1-14 (1991).

30. Iwaki, M., Iwane, A.H., Shimokawa, T., Cooke, R. \& Yanagida, T. Brownian search-and-catch mechanism for myosin-VI steps. Nat. Chem. Biol. 5, 403-405 (2009).

31. Piazzesi, G., Lucii, L. \& Lombardi, V. The size and the speed of the working stroke of muscle myosin and its dependence on the force. J. Physiol. (Lond.) 545, 145-151 (2002).

32. Berg, 0.G., Winter, R.B. \& von Hippel, P.H. Diffusion-driven mechanisms of protein translocation on nucleic acids. 1. Models and theory. Biochemistry 20, 6929-6948 (1981).

33. deCastro, M.J., Fondecave, R.M., Clarke, L.A., Schmidt, C.F. \& Stewart, R.J. Working strokes by single molecules of the kinesin-related microtubule motor ncd. Nat. Cell Biol. 2, 724-729 (2000).

34. Pease, P.J. et al. Sequence-directed DNA translocation by purified FtsK. Science 307, 586-590 (2005).

35. Crozat, E. et al. Separating speed and ability to displace roadblocks during DNA translocation by FtsK. EMBO J. 29, 1423-1433 (2010).

36. Slutsky, M. \& Mirny, L.A. Kinetics of protein-DNA interaction: facilitated target location in sequence-dependent potential. Biophys. J. 87, 4021-4035 (2004).

37. Friedman, L.J. \& Gelles, J. Mechanism of transcription initiation at an activator-dependent promoter defined by single-molecule observation. Cell 148, 679-689 (2012).

38. Skinner, G.M., Baumann, C.G., Quinn, D.M., Molloy, J.E. \& Hoggett, J.G. Promoter binding, initiation, and elongation by bacteriophage T7 RNA polymerase. A single-molecule view of the transcription cycle. J. Biol. Chem. 279, 3239-3244 (2004).

39. Tang, G.Q., Roy, R., Bandwar, R.P., Ha, T. \& Patel, S.S. Real-time observation of the transition from transcription initiation to elongation of the RNA polymerase. Proc. Natl. Acad. Sci. USA 106, 22175-22180 (2009). 


\section{ONLINE METHODS}

Myosin preparation and experiments. Adult male C57 mice were used for this study. Mice were killed by cervical dislocation, and the gastrocnemius muscles were dissected. The experimental protocol for the study of animals was approved by the local animal ethics committee according to the procedures conformed to European Union directive 86/EEC. Myosin was extracted from gastrocnemius muscles (composed of $\sim 100 \%$ type $2 \mathrm{~B}$ myosin $)^{40}$. The S1 subfragment was obtained by proteolytic digestion with papain ${ }^{19}$. G-actin was extracted from rabbit muscle, biotinylated, polymerized to form F-actin filaments and labeled with rhodamine phalloidin ${ }^{19}$. Carboxylated fluorescent beads $(510 \mathrm{~nm}$ diameter, FC03F, Bangs Laboratories) were coated with neutravidin (31000, Pierce) and used in trapping experiments. Smaller beads were used compared to those used in previous studies ${ }^{19}$ to increase velocity in the unbound state $\left(v=F_{\text {tot }} / \gamma\right)$, signal-to-noise ratio and time resolution (Supplementary Notes 1-6). Flow cells were prepared as previously described ${ }^{19}$.

Lac repressor and DNA preparation and experiments. Wildtype lactose repressor was expressed and purified as described previously $^{41}$. A 10.2-kbp DNA construct containing two operator $(\mathrm{O} 1)$ sequences in the center was labeled with biotins at both ends. Briefly, a plasmid containing only two $\mathrm{O} 1$ operators (305 base pairs apart from each other) was digested with PauI (\#ER1091, Fermentas) and SalI (\#ER064, Fermentas). The two restriction sites, which are also close to each other, were located on the opposite side of the plasmid. The two $5^{\prime}$ overhangs created by restriction digestion were filled in by Klenow exo- DNA polymerase (\#EP0421, Fermentas) through the incorporation of biotin-dNTPs (Invitrogen). A custom-made laminar-flow system was used to incubate biological samples, perform buffer exchanges and to efficiently anchor DNA molecules between the two optically trapped beads $(1.87 \mu \mathrm{m}$ streptavidin-coated polystyrene beads, Spherotech). The presence of a single DNA molecule in the dumbbell was assessed by force-extension analysis ${ }^{42}$. LacI (220 nM) was incubated for $3 \mathrm{~min}$ into our multichannel flow cell (previously coated with silica beads and nitrocellulose, as for myosin experiments ${ }^{19}$ ), followed by $3 \mathrm{~min}$ incubation of $1 \mathrm{mg} / \mathrm{ml}$ bovine serum albumin (Sigma). All experiments were performed in $10 \mathrm{mM}$ Tris, $200 \mathrm{mM} \mathrm{KCl}, 0.1 \mathrm{mM}$ EDTA and $0.2 \mathrm{mM}$ DTT.

Experimental apparatus. The experimental setup (Supplementary Fig. 4) comprises an inverted optical microscope combined with double optical tweezers and an apparatus for the detection of single-molecule fluorescence. The sample can be positioned through piezo translators $(x-y$ and $z$ ) with nanometer accuracy and imaged on a charge-coupled device (CCD) camera (CCD with image magnification of $200 \times$ ). The apparatus is stabilized to less than $1 \mathrm{~nm}$ with both passive and active stabilization ${ }^{22,23}$. The bead with the myosin or Lacl molecule was used as a landmark to determine the sample coordinates (CCD with image magnification of 2,000x) and correct for thermal drifts and low-frequency noise by moving piezo translators (nanometer-accuracy stabilization feedback in Supplementary Fig. 4). Double optical tweezers were obtained from a single laser source (Nd:YAG 1,064 nm) through polarizing beam-splitter cubes, overlapped on the optical axis of the microscope through dichroic mirror 2 and focused on the sample plane by the objective. Each trap can be moved along $x$ by AODs placed in a plane conjugate to the back focal plane of the objective. After passing through the sample, the trapping laser was extracted from the microscope optical path (dichroic mirror 3), the two trapping beams were separated by a polarizing beamsplitter cube and position of the trapped beads with respect to the trap center $(x)$ was measured using quadrant detector photodiodes (QDPs) placed in a plane conjugate to the back focal plane of the condenser ${ }^{43}$. The force $F$ applied on each bead was measured from the displacement of the bead from the trap center $(x)$ and from a calibration of the trap stiffness $(k)$, as $F=-k x$. Before each experiment, $k$ was calibrated over the entire range of trap positions used during the experiment, with a power spectrum method $^{44}$ (Supplementary Fig. 1). Trap stiffnesses in the range of $0.03-0.14 \mathrm{pN} / \mathrm{nm}$ were used in the experiments. A custom software written in Labview controlled the feedback system and data acquisition. During force clamp, the position of the trap was controlled by the feedback system through the AODs. The trap position was thus obtained from a calibration of the trap position on the sample plane versus the frequency of acoustic wave driving the AOD. Data were acquired at $200-\mathrm{kHz}$ sample rate with a data acquisition and generation board (NI-PCI-7830R), provided with a field-programmable gate array. For each data point acquired, the variation of the force from the clamped value and the correction to be applied to the traps position were calculated onboard, using the field-programmable gate array and a proportional integral derivative algorithm. Correction signals were sent to two custom-built direct digital synthesizers driving the AODs ${ }^{45}$. The measured feedback delay was $\sim 8 \mu \mathrm{s}$. This delay comprises the time between correction signals are sent from the computer board to the direct digital synthesizers, plus the time taken to move the traps by the AODs, plus the rise time (10-90\%) of the QDP position signals.

Detection of events. Actin-myosin interactions under zero force (Supplementary Fig. 2) were detected from noise reduction of the bead-position signal ${ }^{24}$. Recorded data were analyzed by fitting the signal variance with a two-state function, using a hidden Markov algorithm ${ }^{17}$. This allowed the separation of low-variance states (bound states) from high-variance states (unbound states). The window width used to calculate the signal variance was chosen according to ref. 17, to result in zero or few false detected events. Windows used in the experiments varied from $5 \mathrm{~ms}$ to $10 \mathrm{~ms}$, depending on the signal-to-noise ratio. Therefore, events shorter than $5 \mathrm{~ms}$ (dead time) were not detectable using this configuration. Hidden Markov analysis of dumbbell velocity could not be used to analyze force-clamp experiments. In fact, the kinetics of the myosin-actin and LacI-DNA interactions were not known a priori because of the increased time resolution of the method and the variable load. Moreover, data analysis was complicated by the fact that the signal-to-noise ratio of data increased with the applied force (Supplementary Note 5). We therefore used a different approach, inspired by the work in refs. 46,47, and assuring nearly zero false event detection $(<1 \%)$ under all conditions of our experiments. Briefly, from the position signal of the trap displaying the best signal-to-noise ratio, we calculated the pointby-point velocity. A Gaussian filter with a s.d. $(\sigma)$ was used to smooth the velocity data. Distribution of the smoothed velocity was then fitted with a double Gaussian function (Fig. 2c,d). 
From the fit parameters we calculated an optimal threshold to separate bound and unbound events, such that the probability per unit time of threshold crossing owing to noise from bound and unbound state was the same (Supplementary Note 2). Then, we calculated the number of false events $N_{\text {false }}$ introduced by the analysis (Supplementary Note 2). If $N_{\text {false }}$ was higher than $1 \%$ of the total number of events, we increased the filter width $\sigma$ and repeated the procedure until $N_{\text {false }}$ was $\sim 1 \%$. Values of $\sigma$ used varied between $\sim 1 \mathrm{~ms}$ at low forces $(\sim 1 \mathrm{pN})$ and $\sim 25 \mu \mathrm{s}$ at high forces $(\sim 10 \mathrm{pN})$ because of the increasing signal-to-noise ratio with force. The Gaussian distribution of the velocity in the bound state was narrower than in the unbound state because the stiffness of the system is higher when myosin binds to the actin filament or when LacI binds to DNA. Therefore, the optimal threshold calculated as explained above, is closer to the bound-state velocity (Fig. 2c,d). For this reason, time of the beginning and the end of each interaction were corrected taking into account that the threshold was not halfway between the centers of the two distributions. The times of the beginning and the end were also corrected for very short events (Supplementary Notes 3,4).

Cumulative frequency distributions. Cumulative frequency distributions of the durations of bound events were fitted with a single, double or triple exponential function, which took into account the fraction of lost events, $A_{\text {lost }}$, resulting from limited time resolution. For example, the triple-exponential function takes the form

$$
\begin{gathered}
F(t)=A_{1}\left(1-\exp \left(-k_{1} \times t\right)\right)+A_{2}\left(1-\exp \left(-k_{2} \times t\right)\right) \\
+A_{3}\left(1-\exp \left(-k_{3} \times t\right)\right)-A_{\text {lost }}
\end{gathered}
$$

Ensemble averages. To calculate ensemble averages, events were aligned at their beginning (along the horizontal time axis) and translated (along the vertical position axis) to overlap the position signal in the part preceding binding, where the dumbbell is moving at constant velocity. To this end, we calculated linear regressions of the rising or decreasing part before each event; events were thus superimposed along $x$ and $y$ at the point of intersection of the linear fit with the beginning of the event. To obtain the amplitude of the myosin working stroke, ensemble averages were fitted with a function composed by a linear part before binding and a double exponential after binding (to take into account the two steps of myosin ${ }^{19}$ ). We explain the details in Supplementary Note 7. In case of the actin-myosin interaction, to calculate ensemble averages separately for events belonging to each of the three populations, we first fitted for each recording the cumulative frequency distribution of bound states durations. From the fit parameters, we calculated the durations as follows:

$$
\begin{aligned}
& t_{k_{1} k_{2}}=\ln \left(100 \frac{A_{2}}{A_{1}}\right) /\left(k_{2}-k_{1}\right) \\
& t_{k_{3} k_{2}}=\ln \left(100 \frac{A_{2}}{A_{3}}\right) /\left(k_{2}-k_{3}\right) \\
& t_{k_{1} k_{3}}=\ln \left(100 \frac{A_{3}}{A_{1}}\right) /\left(k_{3}-k_{1}\right)
\end{aligned}
$$

The ratio $N_{1} / N_{2}$ between the number of events belonging to pathway 1 and 2 for durations higher than $t_{k_{1} k_{2}}$ is 100 (Supplementary Note 8). Similarly, $N_{3} / N_{2}$ for durations higher than $t_{k_{3} k_{2}}$ and $N_{1} / N_{3}$ for durations higher than $t_{k_{1} k_{3}}$ were 100 . To calculate ensemble averages excluding very short events (pathway 2), we thus averaged events with durations longer than $\max \left\{t_{k_{1} k_{2}}, t_{k_{3} k_{2}}\right\}$. To calculate ensemble averages including long events only (pathway 1 ), we averaged events with durations longer than $\max \left\{t_{k_{1} k_{2}}, t_{k_{1} k_{3}}\right\}$ (Supplementary Note 8).

40. Canepari, M. et al. Functional diversity between orthologous myosins with minimal sequence diversity. J. Muscle Res. Cell Motil. 21, 375-382 (2000).

41. Zhan, H., Swint-Kruse, L. \& Matthews, K.S. Extrinsic interactions dominate helical propensity in coupled binding and folding of the lactose repressor protein hinge helix. Biochemistry 45, 5896-5906 (2006).

42. Smith, S.B., Cui, Y. \& Bustamante, C. Overstretching B-DNA: the elastic response of individual double-stranded and single-stranded DNA molecules. Science 271, 795-799 (1996).

43. Capitanio, M., Normanno, D. \& Pavone, F.S. High-precision measurements of light-induced torque on absorbing microspheres. Opt. Lett. 29, 2231-2233 (2004).

44. Capitanio, M. et al. Calibration of optical tweezers with differential interference contrast signals. Rev. Sci. Instrum. 73, 1687-1696 (2002).

45. Capitanio, M., Cicchi, R. \& Pavone, F.S. Continuous and time-shared multiple optical tweezers for the study of single motor proteins. Opt. Lasers Eng. 45, 450-457 (2007).

46. Colquhoun, D. \& Sigworth, F.J. Single-Channel Recording (Plenum Press, New York, 1983).

47. Vanzi, F., Sacconi, L. \& Pavone, F.S. Analysis of kinetics in noisy systems: application to single molecule tethered particle motion. Biophys. J. 93, 21-36 (2007). 